\title{
Expression Levels of PIWI-interacting RNA, piR-823, Are Deregulated in Tumor Tissue, Blood Serum and Urine of Patients with Renal Cell Carcinoma
}

\author{
ROBERT ILIEV $^{1,2}$, MICHAL FEDORKO ${ }^{3}$, TANA MACHACKOVA ${ }^{1}$, HANA MLCOCHOVA $^{1}$, MAREK SVOBODA $^{1,2}$, \\ DALIBOR PACIK ${ }^{3}$, JAN DOLEZEL $^{4}$, MICHAL STANIK $^{4}$ and ONDREJ SLABY ST $^{1,2}$ \\ ${ }^{1}$ Central European Institute of Technology, Masaryk University, Brno, Czech Republic; \\ Departments of ${ }^{2}$ Comprehensive Cancer Care, and ${ }^{4}$ Urologic Oncology, \\ Masaryk Memorial Cancer Institute, Brno, Czech Republic; \\ ${ }^{3}$ Department of Urology, University Hospital Brno, Masaryk University, Brno, Czech Republic
}

\begin{abstract}
Background: Renal cell carcinoma (RCC) is the most common neoplasm of adult kidney accounting for about $3 \%$ of adult malignancies. P-Element induced wimpy testis (PIWI)-interacting RNAs (piRNAs) are a new class of naturally occurring, short non-coding RNAs involved in silencing of transposable elements and in sequence-specific chromatin modifications. There were preliminary data published indicating that piR-823 expression is deregulated in circulating tumor cells and tumor tissue in gastric and kidney cancer, respectively. Patients and Methods: In our study, we analyzed piR-823 levels in 588 biological specimens: tumor tissue $(N=153)$, adjacent renal parenchyma $(N=121)$, blood serum $(N=178)$ and urine $(N=20)$ of patients undergoing nephrectomy for RCC; and in blood serum $(N=101)$ and urine $(N=15)$ of matched healthy controls. Expression levels of piR823 were determined in all biological specimens by quantitative real-time polymerase chain reaction, compared in patients and controls, and correlated with clinicopathological features of RCC. Results: We identified a significant downregulation of piR-823 in tumor tissue $[p<0.0001$, area under the curve $(A U C)=0.7945]$. On the contrary in blood serum and urine, the expression of piR-823 was significantly higher in patients with RCC compared to healthy individuals ( $p=0.0005$,
\end{abstract}

Correspondence to: Associate Professor Ondrej Slaby, Ph.D., Central European Institute of Technology (CEITEC), Masaryk University, University Campus Bohunice, Building A35, Room 217, Kamenice 5, 62500 Brno, Czech Republic. Tel: +420 776494155, email: on.slaby@gmail.com and Michal Fedorko, MD, Department of Urology, University Hospital Brno, Jihlavska 340/20, 62500 Brno, Czech Republic. Tel: +420 532231111, e-mail: fedorko.michal@fnbrno.cz

Key Words: PIWI-interacting RNA, piR-823, renal cell carcinoma; tumor tissue, blood serum, urine.
$A U C=0.6264$ and $p=0.0157, A U C=0.7433$, respectively). We further observed higher levels of piR-823 in tumor tissue to be associated with shorter disease-free survival of patients $(p=0.0186)$ and a trend for higher piR-823 levels in serum to be associated with advanced clinical stages of RCC $(p=0.0691)$. There were no other significant associations of piR-823 levels in any type of biological specimen with clinicopathological features of RCC. Conclusion: piR-823 is down-regulated in tumor tissue, but positively correlated with worse outcome, indicating its complex role in RCC pathogenesis. In blood serum, piR-823 is up-regulated, but with unsatisfactory analytical performance. Preliminary data indicate the promising diagnostic utility of urinary piR-823 in patients with RCC.

Renal cell carcinoma (RCC) is the most common neoplasm of the kidney in adults, accounting for $3 \%$ of adult malignancies and having the highest mortality rate at over $40 \%$ (1). P-Element induced wimpy testis (PIWI)-interacting RNAs (piRNAs) are a newly discovered class of short noncoding RNAs. They are 26-31 nucleotides long, which clearly differentiate them from other short non-coding RNAs such as microRNAs and siRNAs with 20-23 nucleotides in length (2). Initially, they were reported in mice testes, where they have a major role in the germ cell in maintaining genomic stability through binding to PIWI proteins and then silencing transposable elements (3). piRNA-based silencing is mediated by $\mathrm{CpG}$ methylation, chromatin remodeling and degradation of complementary transcripts (4). Recent studies suggest that the deregulated expression of PIWI proteins is common in tumor tissue, and PIWI expression levels are correlated to clinicopathological features and survival in patients with cancer [reviewed in (5)]. Therefore, they present promising cancer biomarkers. Recently, it was repeatedly reported that not only PIWI proteins, but also 
Table I. Patient characteristics and piR-823 expression levels.

\begin{tabular}{|c|c|c|c|c|c|c|}
\hline & \multicolumn{2}{|c|}{ Tissue } & \multicolumn{2}{|c|}{ Serum } & \multicolumn{2}{|c|}{ Urine } \\
\hline & $\mathrm{N}$ & piR-823 copies* & $\mathrm{N}$ & piR-823 copies* & $\mathrm{N}$ & piR-823 copies* \\
\hline Parenchyma, HC & 121 & $465528 \pm 391450$ & 101 & $2427 \pm 4230$ & 15 & $93200 \pm 80495$ \\
\hline Tumor, RCC patients & 153 & $161841 \pm 191214$ & 178 & $3828 \pm 6433$ & 20 & $552264 \pm 773929$ \\
\hline Fold-change in patients & & 0.3477 & & 1.5772 & & 4.663 \\
\hline AUC & & 0.7945 & & 0.6264 & & 0.7433 \\
\hline$p$-Value & & 0.0001 & & 0.0005 & & 0.0157 \\
\hline \multicolumn{7}{|l|}{ TNM stage } \\
\hline I-II & 82 & $201075 \pm 314067$ & 117 & $2422 \pm 2823$ & & NA \\
\hline III-IV & 48 & $151862 \pm 129437$ & 60 & $3634 \pm 6378$ & & NA \\
\hline$p$-Value & & 0.7852 & & 0.0691 & & NA \\
\hline \multicolumn{7}{|l|}{ Fuhrman grade } \\
\hline G1-2 & 81 & $213445 \pm 316014$ & 106 & $2391 \pm 2601$ & & NA \\
\hline G3-4 & 49 & $135291 \pm 115428$ & 66 & $3621 \pm 6326$ & & NA \\
\hline$p$-Value & & 0.1416 & & 0.2096 & & NA \\
\hline \multicolumn{7}{|l|}{ Association with DFS } \\
\hline Cut-off & & 223038 & & 2046 & & NA \\
\hline$p$-Value & & 0.0186 & & 0.9180 & & NA \\
\hline \multicolumn{7}{|l|}{ Association with OS } \\
\hline Cut-off & & 147727 & & 2046 & & NA \\
\hline$p$-Value & & 0.6042 & & 0.9035 & & NA \\
\hline
\end{tabular}

RCC, Renal cell carcinoma; TU, tumor; RP, renal parenchyma; HC, healthy controls; AUC, area under curve; DFS, disease-free survival; OS, overall survival; NA: not available. ${ }^{*}$ Data are the mean \pm SD.

piRNAs can play an important role in carcinogenesis. Overor underexpression of piRNAs that target mRNA transcripts (e.g. those containing transposon-derived sequences in their 3' untranslated regions) could also play a driver role through degradation or inhibition of tumor-suppressor genes or oncogenes, respectively. Deregulated levels of many piRNAs were described in $\operatorname{RCC}(6,7)$, gastric cancer $(8,9)$, hepatocellular carcinoma (10), multiple myeloma (11), lung cancer (12), bladder cancer (13), breast cancer (14) and pancreatic ductal adenocarcinoma (15). One of the first piRNAs identified as being linked to cancer is piR-823 (8). piR-823 was detected in cancer cell lines, white blood cells (16) and blood plasma $(17,18)$. This piRNA was described to be deregulated in gastric tumor tissue and myeloma cells and to be involved in regulation of tumor cell growth $(8,11)$. We published preliminary reports indicating its downregulation in RCC tissue (19). Here, we decided to perform large-scale evaluation of piR-823 deregulation in tumor tissue, blood serum and urine of patients with RCC.

\section{Patients and Methods}

Samples of tumor tissue $(\mathrm{N}=153)$, renal parenchyma $(\mathrm{N}=121)$, blood serum $(\mathrm{N}=178)$ and urine (only for the last 20 patients included) used in this study were obtained from patients diagnosed with RCC who underwent radical nephrectomy at Masaryk Memorial Cancer Institute (MMCI) in Brno or The University Hospital Brno (UHB) between 2012 and 2015. The median age of the patients at the time of diagnosis was 64 years (range $=21-84$ years). Blood serum $(\mathrm{N}=101)$ and urine samples $(\mathrm{N}=15)$ were also obtained from healthy individuals who underwent preventative medical examination in MMCI. Patient's biological samples were frozen and stored at $-80^{\circ} \mathrm{C}$ until processing. Written informed consent was obtained from all participants (patients, healthy individuals), and the study was approved by the local Ethics Boards at MMCI and UHB. Clinical and pathological characteristics are summarized in Table I.

Total RNA from tissue samples was isolated by use of mirVana miRNA Isolation Kit (Life Technologies, Carlsbad, CA, USA) according to the manufacturer's instructions. For RNA isolation from serum, miRNeasy Serum/Plasma Kit (Qiagen, Valencia, CA, USA) was used, and for isolation from urine Urine microRNA Purification Kit (Norgen, Thorold, ON, Canada) was used. RNA concentration and purity were measured using a NanoDrop 1000 spectrophotometer (Thermo Fisher Scientific, Waltham, MA, USA). Using $10 \mathrm{ng}$ of total RNA, cDNA was synthesized with TaqMan ${ }^{\circledR}$ MicroRNA Reverse Transcription Kit (Thermo Fisher Scientific) according to the manufacturer's recommendations. Quantitative polymerase chain reaction was performed in a total volume of $15 \mu \mathrm{l}$ using specific probe for $p i R-823$. The primers and probe were designed and synthesized (Thermo Fisher Scientific) to be used for determination of piR-823 sequence 5' AGCGUUGGUGGUAUAGUGGUGAGC AUAGCUGC-3'. The expression levels were quantified absolutely by use of a calibration curve using piR- 823 plasmids synthesized by Generi Biotech (Hradec Kralove, Czech Republic).

Differences in expression levels of piR-823 between tumor tissue and renal parenchyma were evaluated by use of Wilcoxon test, and differences in serum and urine samples of patients and healthy controls were evaluated by use of Mann-Whitney test. For determining the differences according to clinical stage and grade, we used non- 


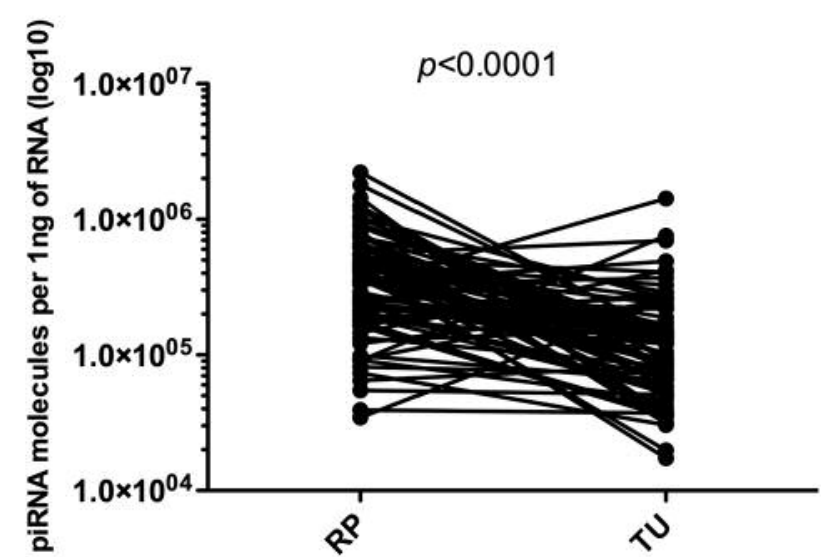

Figure 1. Statistical significant difference in piR-823 expression $(p<0.0001)$ between 121 paired samples of renal cell carcinoma tumor tissue $(T U)$ and paired healthy renal parenchyma $(R P)$.

parametric Kruskal-Wallis test. Receiver operating curve (ROC) analysis was performed to identify cut-offs to distinguish patients with different prognoses. Kaplan-Meier survival curves and log-rank test were used for survival analysis. Calculations were performed in GraphPad Prism 6 (GraphPad Software, Inc., La Jolla, CA, USA).

\section{Results}

In our study, we successfully determined $p i R-823$ expression levels in 588 biological specimens obtained from patients with RCC and healthy controls. We observed significant differences in piR-823 expression levels between tumor and non-tumor tissue samples. piR-823 expression was significantly decreased in tumor tissue compared to paired non-tumorous renal parenchyma (Figure $1, p<0.0001$ ). ROC analysis showed that tissue levels of piR-823 can differentiate tumor tissue from renal parenchyma with an area under the curve (AUC) of 0.795. However, we observed no association of piR-823 levels in tumor with clinical stage or Fuhrmann grade. Interestingly, although the piR823 levels in tumor tissue were decreased, a low level was associated with a longer disease-free survival (DFS) $(p=0.0186$; Figure $2)$. The median DFS in patients with RCC with high expression of piR-823 was 56 months, whereas in the lowexpression group median was not reached. Although we observed the same trend in OS, the association of $p i R-823$ level and OS did not reach statistical significance $(p>0.05)$.

Furthermore, we recorded significantly higher levels of piR-823 in serum of patients with RCC when compared to healthy controls ( $p=0.0005$; Figure $3 \mathrm{~A})$, but ROC analysis showed this to have unsatisfactory analytical performance in distinguishing patients with RCC from controls (AUC $=0.626$ ). There was no correlation of $p i R-823$ in serum with Furhmann grade, DFS or OS. We did observe a trend

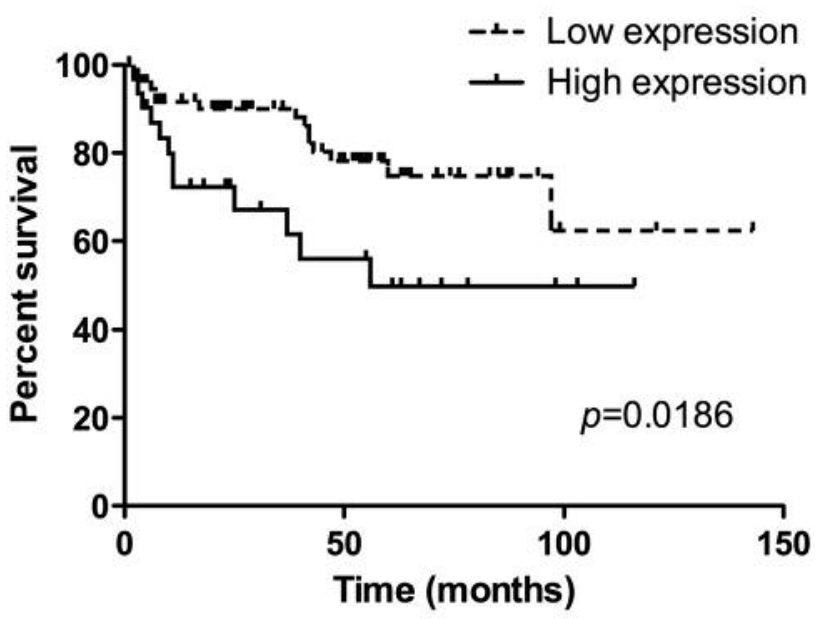

Figure 2. piR-823 levels and disease-free survival of patients with renal cell carcinoma. There was a proven statistically significant $(p=0.0186)$ association of longer disease-free survival in patients with low expression of piR-823 in tumor tissue compared to those with high expression.

for higher $p i R-823$ levels in serum to be associated with advanced clinical stages of RCC $(p=0.0691)$. There was also no correlation between the piR-823 level in serum and that in paired tumor tissues samples $(p>0.05)$.

Finally, we determined level of piR-823 in urinary samples of small groups of patients with RCC and healthy controls. Preliminary results showed $p i R-823$ levels were significantly higher in urinary samples from patients with RCC in comparison to healthy controls ( $p=0.0157$, Figure 3B).

\section{Discussion}

As the first aim, we determined expression of piR-823 in paired samples of tumor tissue and adjacent renal parenchyma. In agreement with our previous pilot study (19), we observed significantly reduced expression of piR-823 in tumor tissue compared to adjacent non-tumorous renal parenchyma. Martinez et al.'s observed RCC tumors to be characterized by general down-regulation of piRNA (9). We did not find any significant association of tumor piR-823 level with clinical stage, Fuhrmann grade or OS. However, we observed significant association of tumor piR-823 and DFS, with the median DFS in patients with low expression being significantly longer.

Since piRNAs predominantly control the expression of transposable elements (20), piR-823 could be involved in maintenance of genomic stability and its frequently observed loss in RCC tumor tissues may be linked to genomic instability, which is a general hallmark of all malignant tumors. However, the link we found here to survival, where a higher levels of piR-823 is associated with worse DFS, indicates a more oncogenic than tumor-suppressive role for 

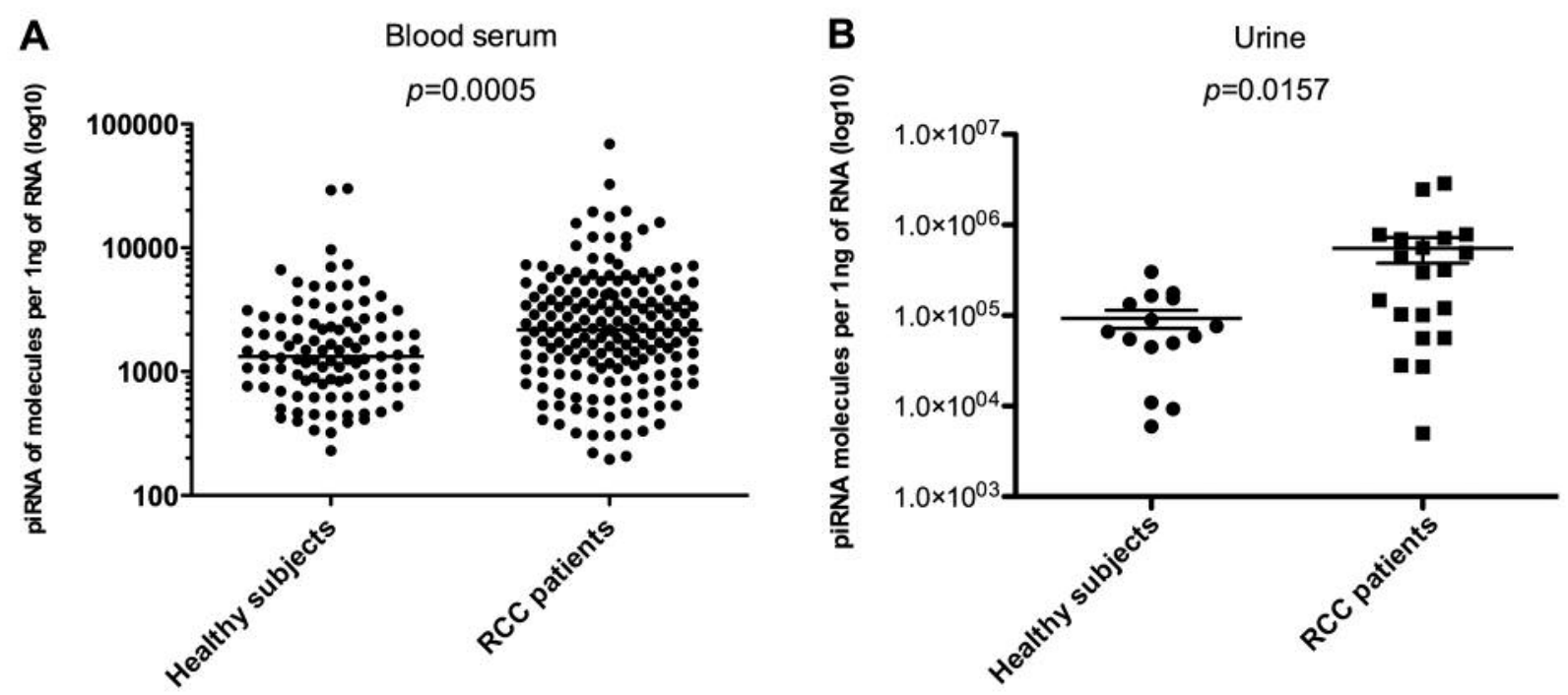

Figure 3. piR-823 levels measured in blood serum (A) and urine (B). In patients with renal cell carcinoma (RCC), the piR-823 levels were increased compared to those in healthy individuals. Levels of piR-823 in urine of patients with RCC were higher in comparison with those in urine of healthy individuals. Lines represent medians, and bars are 25 th and 75 th percentiles.

piR-823 in RCC. It seems that piR823 might play a complex role in RCC pathogenesis characterized by different phenotypic effects in different stages of the disease.

In addition to transposable elements, piR-823 can potentially also target protein-coding genes. On the basis of base pair complementarity, cell-division cycle 5-like protein (CDC5L) represents a potential target of piR-823, since CDC5L contains a region which is fully complementary to piR-823. CDC5L is a cell-cycle regulator (21) whose its up-regulation in tumor tissue of osteosarcoma and cervical tumors led to aberrant cellcycle control and contributed to the malignant phenotype (22). CDC5L is a part of the E3 ubiquitin ligase complex and is required for the correct functioning of the S-phase cell-cycle checkpoint (23). It is also involved in RNA processing, where it plays an important role as a pre-mRNA splicing factor and its down-regulation reduces cell viability (22). Unfortunately, the role of CDC5L in RCC is not known.

We also analyzed piR-823 levels in blood serum and urine. We determined piR-823 expression levels in a large group of patients with RCC and gender- and age-matched healthy controls. We observed significantly higher serum levels of piR-823 in the group of patients compared to healthy individuals $(p=0.0005)$, which is in contrast to differences we found in tissue samples. This phenomenon might be explained by active release of piR-823 by tumor cells leading to its decreased levels in tumors and increased levels in the circulation. The AUC for the ability of serum piR823 to distinguish patients with RCC from healthy controls was 0.626 , which is not high enough to enable diagnostic utility. Although differences in expression does not allow usage of piR-823 as a new independent biomarker of RCC, it could potentially be useful in combination with other available biomarkers, e.g. newly described microRNA biomarkers. We did not observe any significant association of $p i R-823$ with any clinical stage, Furhmann grade or survival. Therefore, we suggest that $p i R-823$ is more likely linked to some of the general hallmarks of RCC pathogenesis than to the tumor features behind tumor invasion and progression of the disease.

Finally, we evaluated piR-823 levels in urine samples of pilot cohort of 20 RCC patients and 15 healthy controls. By this analysis, we identified significantly higher levels of piR823 in urine from patients and significantly higher levels of piR-823 in urine in comparison to serum, indicating urine to be more suitable for $p i R-823$ analysis than serum. In order to evaluate potential associations of urinary piR-823 and clinicopathological data, study on the larger cohort of patients with RCC is needed.

There are certain limitations to our study. We performed absolute quantification of piR-823 as we were not able to identify an appropriate endogenous control to be used for relative quantification. Therefore, our data might be biased due to the presence of inhibitors in the complex biological specimens. Moreover, in vitro experiments are needed to demonstrate the ability of RCC cells to release $p i R-823$ and to evaluate whether $p i R-823$ is exosomal, protein-bound or free. We have not performed an in vitro and in vivo study to describe functioning of piR-823 in RCC pathogenesis.

In conclusion, piR-823 is down-regulated in tumor tissue, but in tumor positively correlated with worse outcome, indicating its complex role in RCC pathogenesis. In blood serum, piR-823 is up-regulated, but with unsatisfactory 
analytical performance. Preliminary data indicate the promising diagnostic utility of urinary piR-823 in patients with RCC

\section{Disclosure Statement}

The Authors have no conflict of interest.

\section{Acknowledgements}

This work was financially supported by the Czech Ministry of Health, grant no. 15-34678A, 15-31071A, project MZ CR - RVO (MOU, 00209805), by the Ministry of Education, Youth and Sports of the Czech Republic under the project CEITEC 2020 (LQ1601) and project BBMRI CZ (LM2015089).

\section{References}

1 Albiges L, Fay AP, McKay RR, Kaymakcalan MD and Choueiri TK: Diagnosis of renal cell carcinoma: a clinician's perspective. Surg Pathol Clin 8: 657-662, 2015.

2 Aravin A, Gaidatzis D, Pfeffer S, Lagos-Quintana M, Landgraf P, Iovino N, Morris P, Brownstein MJ, Kuramochi-Miyagawa S, Nakano T, Chien M, Russo JJ, Ju J, Sheridan R, Sander C, Zavolan $\mathrm{M}$ and Tuschl T: A novel class of small RNAs bind to MILI protein in mouse testes. Nature 442: 203-207, 2006.

3 Brennecke J, Aravin AA, Stark A, Dus M, Kellis M, Sachidanandam R and Hannon GJ: Discrete small RNAgenerating loci as master regulators of transposon activity in Drosophila. Cell 128: 1089-1103, 2007.

4 Guo M and Wu Y: Fighting an old war with a new weapon silencing transposons by Piwi-interacting RNA. IUBMB Life 65: 739-747, 2013.

5 Suzuki R, Honda S and Kirino Y. PIWI Expression and Function in Cancer. Front Genet 3: 204, 2012.

6 Busch J, Ralla B, Jung M, Wotschofsky Z, Trujillo-Arribas E, Schwabe P, Kilic E, Fendler A and Jung K: Piwi-interacting RNAs as novel prognostic markers in clear cell renal cell carcinomas. J Exp Clin Cancer Res 34: 61, 2015.

7 Li Y, Wu X, Gao H, Jin JM, Li AX, Kim YS, Pal SK, Nelson RA, Lau CM, Guo C, Mu B, Wang J, Wang F, Wang J, Zhao Y, Chen W, Rossi JJ, Weiss LM and Wu H: Piwi-Interacting RNAs (piRNAs) Are Dysregulated in Renal Cell Carcinoma and Associated with Tumor Metastasis and Cancer-Specific Survival. Mol Med 21: 381-388, 2015.

8 Cheng J, Deng H, Xiao B, Zhou H, Zhou F, Shen Z and Guo J: piR-823, a novel non-coding small RNA, demonstrates in vitro and in vivo tumor suppressive activity in human gastric cancer cells. Cancer Lett 315: 12-17, 2012.

9 Martinez VD, Vucic EA, Thu KL, Hubaux R, Enfield KS, Pikor LA, Becker-Santos DD, Brown CJ, Lam S and Lam WL: Unique somatic and malignant expression patterns implicate PIWIinteracting RNAs in cancer-type specific biology. Sci Rep 5: 10423, 2015.

10 Law PT, Qin H, Ching AK, Lai KP, Co NN, He M, Lung RW, Chan AW, Chan TF and Wong N: Deep sequencing of small RNA transcriptome reveals novel non-coding RNAs in hepatocellular carcinoma. J Hepatol 58: 1165-1173, 2013.

11 Yan H, Wu QL, Sun CY, Ai LS, Deng J, Zhang L, Chen L, Chu ZB, Tang B, Wang K, Wu XF, Xu J and Hu Y: piRNA-823 contributes to tumorigenesis by regulating de novo DNA methylation and angiogenesis in multiple myeloma. Leukemia 29: 196-206, 2015.

12 Mei Y, Wang Y, Kumari P, Shetty AC, Clark D, Gable T, MacKerell AD, Ma MZ, Weber DJ, Yang AJ, Edelman MJ and Mao L: A piRNA-like small RNA interacts with and modulates p-ERM proteins in human somatic cells. Nat Commun 6: 7316, 2015.

13 Chu H, Hui G, Yuan L, Shi D, Wang Y, Du M, Zhong D, Ma L, Tong N, Qin C, Yin C, Zhang Z and Wang M: Identification of novel piRNAs in bladder cancer. Cancer Lett 356: 561-677, 2015.

14 Hashim A, Rizzo F, Marchese G, Ravo M, Tarallo R, Nassa G, Giurato G, Santamaria G, Cordella A, Cantarella C and Weisz A: RNA sequencing identifies specific PIWI-interacting small non-coding RNA expression patterns in breast cancer. Oncotarget 5: 9901-9910, 2014.

15 Müller S, Raulefs S, Bruns P, Afonso-Grunz F, Plötner A, Thermann R, Jäger C, Schlitter AM, Kong B, Regel I, Roth WK, Rotter B, Hoffmeier K, Kahl G, Koch I, Theis FJ, Kleeff J, Winter $\mathrm{P}$ and Michalski CW: Next-generation sequencing reveals novel differentially regulated mRNAs, IncRNAs, miRNAs, sdRNAs and a piRNA in pancreatic cancer. Mol Cancer 14: 94, 2015.

16 Cui L, Lou Y, Zhang X, Zhou H, Deng H, Song H, Yu X, Xiao B, Wang W and Guo J: Detection of circulating tumor cells in peripheral blood from patients with gastric cancer using piRNAs as markers. Clin Biochem 13: 1050-1057, 2011.

17 Yang X, Cheng Y, Lu Q, Wei J, Yang H and Gu M: Detection of stably expressed piRNAs in human blood. Int J Clin Exp Med 8: 13353-13358, 2015.

18 Freedman JE, Gerstein M, Mick E, Rozowsky J, Levy D, Kitchen R, Das S, Shah R, Danielson K, Beaulieu L, Navarro FC, Wang Y, Galeev TR, Holman A, Kwong RY, Murthy V, Tanriverdi SE, Koupenova-Zamor M, Mikhalev E and Tanriverdi $\mathrm{K}$ : Diverse human extracellular RNAs are widely detected in human plasma. Nat Commun 7: 11106, 2016.

19 Iliev R, Stanik M, Fedorko M, Poprach A, VychytilovaFaltejskova P, Slaba K, Svoboda M, Fabian P, Pacik D, Dolezel J and Slaby O: Decreased expression levels of PIWIL1, PIWIL2, and PIWIL4 are associated with worse survival in renal cell carcinoma patients. Onco Targets Ther 9: 217-222, 2016.

20 Klattenhoff C, Xi H, Li C, Lee S, Xu J, Khurana JS, Zhang F, Schultz N, Koppetsch BS, Nowosielska A, Seitz H, Zamore PD, Weng $\mathrm{Z}$ and Theurkauf WE: The Drosophila HP1 homolog Rhino is required for transposon silencing and piRNA production by dual-strand clusters. Cell 138: 1137-1149, 2009.

21 Lu XY, Lu Y, Zhao YJ, Jaeweon K, Kang J, Xiao-Nan L, Ge G, Meyer R, Perlaky L, Hicks J, Chintagumpala M, Cai WW, Ladanyi M, Gorlick R, Lau CC, Pati D, Sheldon M and Rao PH: Cell cycle regulator gene $C D C 5 L$, a potential target for $6 \mathrm{p} 12-\mathrm{p} 21$ amplicon in osteosarcoma. Mol Cancer Res 6: 937-946, 2008.

22 Mu R, Wang YB, Wu M, Yang Y, Song W, Li T, Zhang WN, Tan B, Li AL, Wang N, Xia Q, Gong WL, Wang CG, Zhou T, Guo N, Sang ZH and Li HY: Depletion of pre-mRNA splicing factor Cdc5L inhibits mitotic progression and triggers mitotic catastrophe. Cell Death Dis 5: e1151, 2014.

23 Zhang N, Kaur R, Akhter S and Legerski RJ: CDC5L interacts with ATR and is required for the S-phase cell-cycle checkpoint. EMBO Rep 10: 1029-1035, 2009.

Received September 7, 2016

Revised September 28, 2016

Accepted September 29, 2016 